SLAC-PUB-9492

September 2002

\title{
EFFECTS OF RADION MIXING ON THE STANDARD MODEL HIGGS BOSON
}

\author{
THOMAS G. RIZZO ${ }^{a}$ \\ Stanford Linear Accelerator Center, Stanford, \\ CA 94309, USA
}

\begin{abstract}
We discuss how mixing between the Standard Model Higgs boson and the radion of the Randall-Sundrum model can lead to significant shifts in the expected properties of the Higgs boson. In particular we show that the total and partial decay widths of the Higgs, as well as the $h \rightarrow g g$ branching fraction, can be substantially altered from their SM expectations, while the remaining branching fractions are modified less than about $5 \%$ for most of the parameter space volume. Precision measurements of Higgs boson properties at at a Linear Collider are shown to probe a large region of the Randall-Sundrum model parameter space.
\end{abstract}

The Randall-Sundrum (RS) model ${ }^{1}$ offers a potential solution to the hierarchy problem. In this model the Standard Model (SM) fields lie on one of two branes that are embedded in $A d S_{5}$ described by the metric $d s^{2}=$ $e^{-2 k|y|} \eta_{\mu \nu} d x^{\mu} d x^{\nu}-d y^{2}$, where $k$ is the 5 -d curvature parameter of order the Planck scale. To solve the hierarchy problem the separation between the two branes, $r_{c}$, must have a value of $k r_{c} \sim 11-12$. That this quantity can be stabilized and made natural has been demonstrated by a number of authors and leads directly to the existence of a $\operatorname{radion}(r)$, which corresponds to a quantum excitation of the brane separation. It can be shown that the radion couples to the trace of the stress-energy tensor formed from the SM fields with a strength $\Lambda$ of order the $\mathrm{TeV}$ scale, i.e., $\mathcal{L}_{\text {eff }}=-r T_{\mu}^{\mu} / \Lambda$. This leads to couplings for the radion that are qualitatively similar to those of the SM Higgs boson. The radion mass $\left(m_{r}\right)$, which is generated by the brane separation stabilization mechanism, is expected to be significantly below the scale $\Lambda$ implying that the radion may be the lightest new field predicted by the RS model.

On general grounds of covariance, the radion may mix with the SM Higgs

${ }^{a}$ Work supported by the Department of Energy, Contract DE-AC03-76SF00515 
field through an interaction term of the form

$$
S_{r H}=-\xi \int d^{4} x \sqrt{-g_{w}} R^{(4)}\left[g_{w}\right] H^{\dagger} H
$$

where $H$ is the Higgs doublet field, $R^{(4)}\left[g_{w}\right]$ is the Ricci scalar constructed out of the induced metric $g_{w}$ on the SM brane, and $\xi$ is a dimensionless mixing parameter assumed to be of order unity and with unknown sign. The above action induces kinetic mixing between the 'weak eigenstate' $r_{0}$ and $h_{0}$ fields which can be removed through a set of field redefinitions and rotations. Clearly, since the radion and Higgs boson couplings to other SM fields differ this mixing will induce modifications in the usual SM expectations for the Higgs decay widths and branching fractions.

To make unique predictions in this scenario we need to specify four parameters: the masses of the physical Higgs and radion fields, $m_{h, r}$, the mixing parameter $\xi$ and the ratio $v / \Lambda$, where $v$ is the vacuum expectation value of the SM Higgs $\simeq 246 \mathrm{GeV}$. Clearly the ratio $v / \Lambda$ cannot be too large as $\Lambda$ is already bounded from below by collider and electroweak precision data; for definiteness we will take $v / \Lambda \leq 0.2$ and $-1 \leq \xi \leq 1$. When we require that the weak basis mass-squared parameters of the radion and Higgs fields be real, as is required by hermiticity, we obtain an additional constraints on the ratio of the physical radion and Higgs masses which only depends on the product $|\xi| \frac{v}{\Lambda}$. These constraints are incorporated into the analysis below.
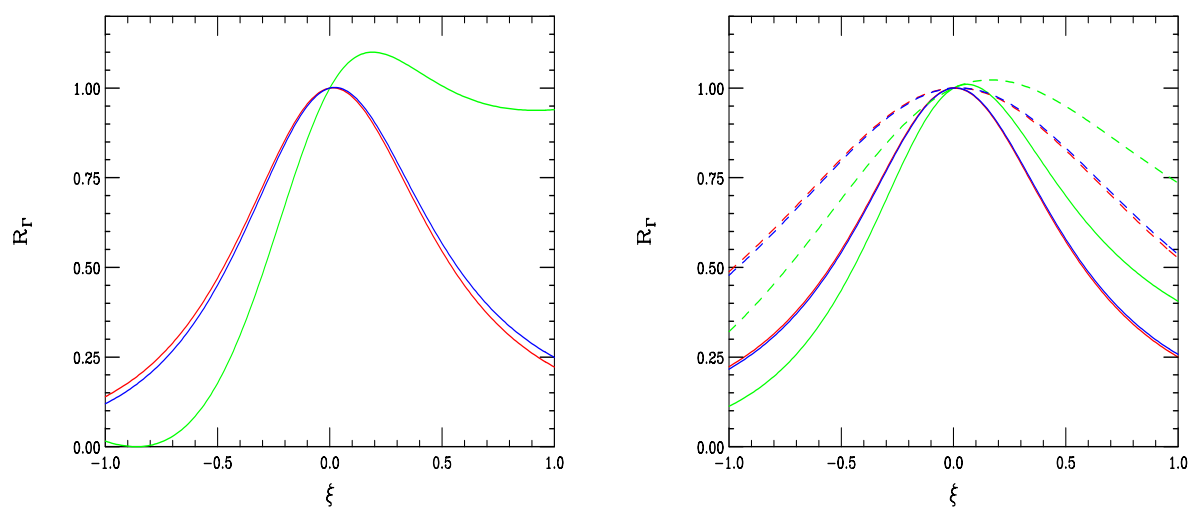

Figure 1: Ratio of Higgs widths to their SM values, $R_{\Gamma}$, as a function of $\xi$ assuming a physical Higgs mass of $125 \mathrm{GeV}$ : red for fermion pairs or massive gauge boson pairs, green for gluons and blue for photons. In the left panel we assume $m_{r}=300 \mathrm{GeV}$ and $v / \Lambda=0.2$. In the right panel the solid(dashed) curves are for $m_{r}=500(300) \mathrm{GeV}$ and $v / \Lambda=0.2(0.1)$. 
Following the notation of Giudice et al., the coupling of the physical Higgs to the SM fermions and massive gauge bosons $V=W, Z$ is given by

$$
\mathcal{L}=\frac{-1}{v}\left(m_{f} \bar{f} f-m_{V}^{2} V_{\mu} V^{\mu}\right)\left[\cos \rho \cos \theta+\frac{v}{\Lambda}(\sin \theta-\sin \rho \cos \theta)\right] h,
$$

where the angle $\rho$ is given above and $\theta$ can be calculated in terms of the parameters $\xi$ and $v / \Lambda$ and the physical Higgs and radion masses. Denoting the combinations $\alpha=\cos \rho \cos \theta$ and $\beta=\sin \theta-\sin \rho \cos \theta$, the corresponding Higgs coupling to gluons can be written as $c_{g} \frac{\alpha_{s}}{8 \pi} G_{\mu \nu} G^{\mu \nu} h$ with $c_{g}=\frac{-1}{2 v}[(\alpha+$ $\left.\left.\frac{v}{\Lambda} \beta\right) F_{g}-2 b_{3} \beta \frac{v}{\Lambda}\right]$ where $b_{3}=7$ is the $S U(3) \beta$-function and $F_{g}$ is a well-known kinematic function of the ratio of masses of the top quark to the physical Higgs. Similarly the physical Higgs couplings to two photons is now given by $c_{\gamma} \frac{\alpha_{e m}}{8 \pi} F_{\mu \nu} F^{\mu \nu} h$ where $c_{\gamma}=\frac{1}{v}\left[\left(b_{2}+b_{Y}\right) \beta \frac{v}{\Lambda}-\left(\alpha+\frac{v}{\Lambda} \beta\right) F_{\gamma}\right]$, where $b_{2}=19 / 6$ and $b_{Y}=-41 / 6$ are the $S U(2) \times U(1) \beta$-functions and $F_{\gamma}$ is another well-known kinematic function of the ratios of the $W$ and top masses to the physical Higgs mass. (Note that in the simultaneous limits $\alpha \rightarrow 1, \beta \rightarrow 0$ we recover the usual SM results.) From these expressions we can now compute the change of the various decay widths and branching fractions of the SM Higgs due to mixing with the radion.

Fig. 1 shows the ratio of the various Higgs widths in comparison to their $\mathrm{SM}$ expectations as functions of the parameter $\xi$ assuming that $m_{h}=125$ $\mathrm{GeV}$ with different values of $m_{r}$ and $\frac{v}{\Lambda}$. We see several features right away: $(i)$ the shifts in the widths to $\bar{f} f / V V$ and $\gamma \gamma$ final states are very similar; this is due to the relatively large magnitude of $F_{\gamma}$ while the combination $b_{2}+b_{Y}$ is rather small. (ii) On the otherhand the shift for the $g g$ final state is quite different since $F_{g}$ is smaller than $F_{\gamma}$ and $b_{3}$ is quite large. (iii) For relatively light radions with a low value of $\Lambda$ the Higgs decay width into the $g g$ final state can come close to vanishing due to a strong destructive interference between the two contributions to the amplitude for values of $\xi$ near -1. (iv) Increasing the value of $m_{r}$ has less of an effect on the width shifts than does a decrease in the ratio $\frac{v}{\Lambda}$.

The deviation from the SM expectations for the various branching fractions, as well as the total width, of the Higgs are displayed in Fig. 2 as a function of the mixing parameter $\xi$. We see that the gluon branching fraction and the total width may be drastically different than that of the SM. As we will see below the former will affect the Higgs production cross section at the LHC. However, the $\gamma \gamma, f \bar{f}$, and $V V$, where $V=W, Z$ branching fractions receive small corrections to their SM values, of order $5-10 \%$ for almost all of the parameter region. Observation of these shifts will require the accurate determination of the Higgs branching fractions obtainable at an $e^{+} e^{-}$Linear 

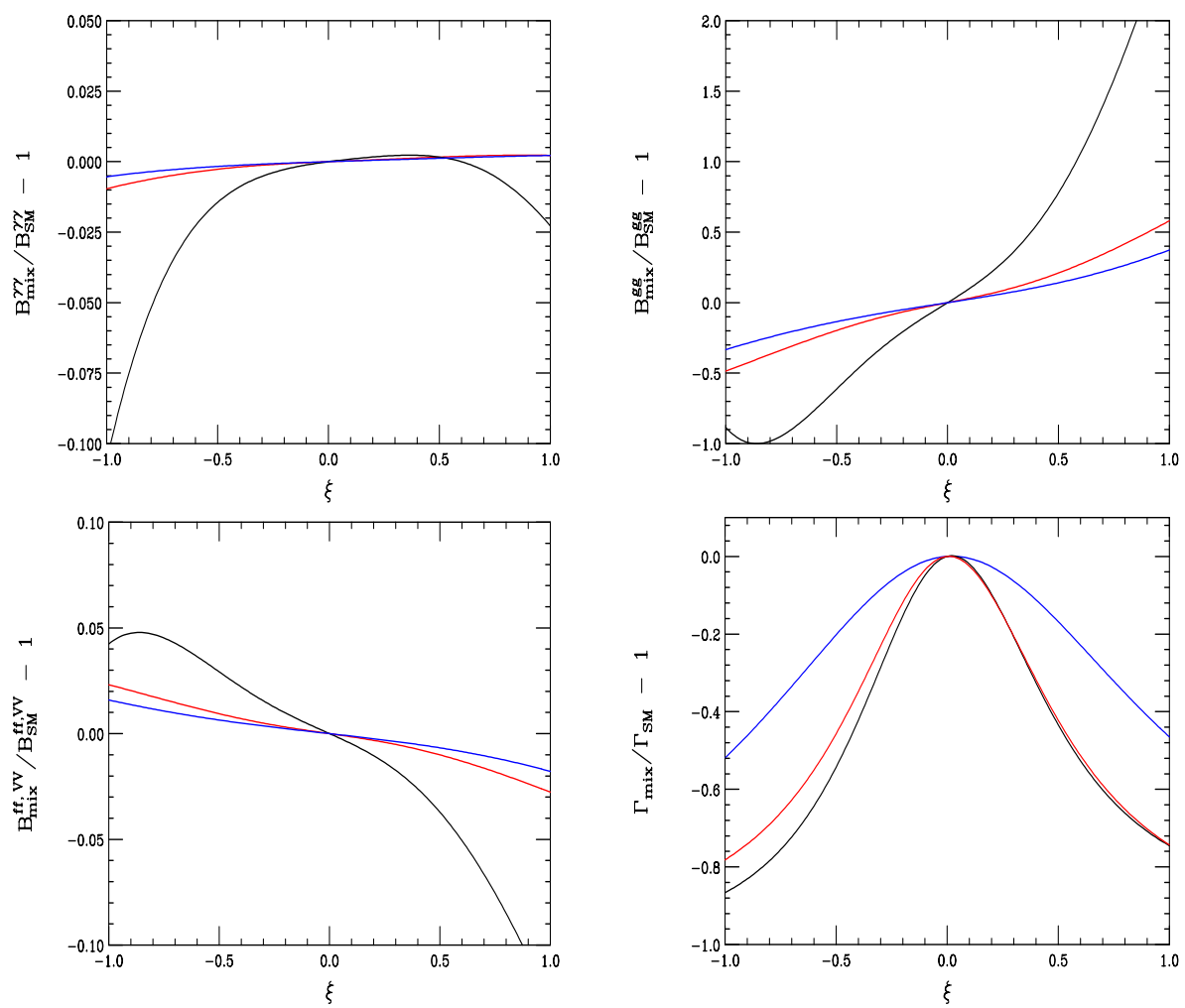

Figure 2: The deviation from the SM expectations for the Higgs branching fraction into $\gamma \gamma$, $g g, f \bar{f}$, and $V V$ final states as labeled, as well as for the total width. The black, red, and blue curves correspond to the parameter choices $m_{r}=300,500,300 \mathrm{GeV}$ with $v / \xi=0.2,0.2,0.1$, respectively. 
Collider (LC) from which constraints on the radion model parameter space may be extracted as will be discussed below. These small changes in the $Z Z h$ and $h b \bar{b}$ couplings of the Higgs boson can also lead to small reductions in the Higgs search reach from LEPII. This is shown in Fig. 3 for several sets of parameters; except for extreme cases this reduction in reach is rather modest.

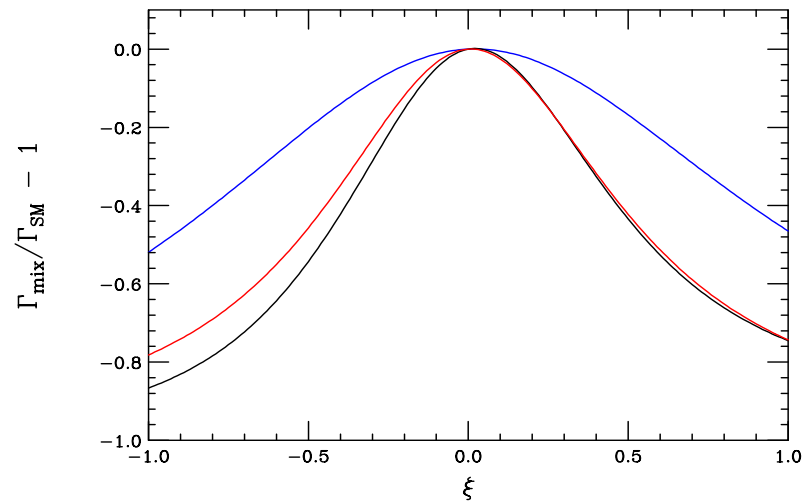

Figure 3: Lower bound on the mass of the Higgs boson from direct searches at LEP as a function of $\xi$ including the effects of mixing. The red (blue; green) curves correspond to the choice $m_{r}=300 \mathrm{GeV}, v / \Lambda=0.2(500,0.2 ; 300,0.1)$.

At the LHC the dominant production mechanism/signal for the light Higgs boson is via the gluon-gluon fusion through a triangle graph with subsequent decay into $\gamma \gamma$. Both the production cross section and the subsequent $\gamma \gamma$ branching fraction are modified by mixing as shown in Fig. 4. This figure shows that the Higgs production rate in this mode at the LHC is always reduced in comparison to the expectations of the SM due to the effects of mixing. For some values of the parameters this reduction can be by more than an order of magnitude which could seriously hinder Higgs discovery via this channel at the LHC.

Once both data from the LHC and LC become available the radion parameter space can be explored using both direct as well as indirect searches. For example, as discussed above, precision measurements of the Higgs boson couplings at these machines can be used to constrain the model parameter space beyond what may be possible through direct searches only. For purposes of demonstration let us assume that the LHC/LC measure these couplings to be consistent with the expectations of the SM. We then can ask what regions in the $\xi-m_{r}$ plane would remain allowed in this case as the ratio $v / \Lambda$ is varied; the results are shown in Fig. 5 assuming a Higgs mass of $125 \mathrm{GeV}$. Here we see 


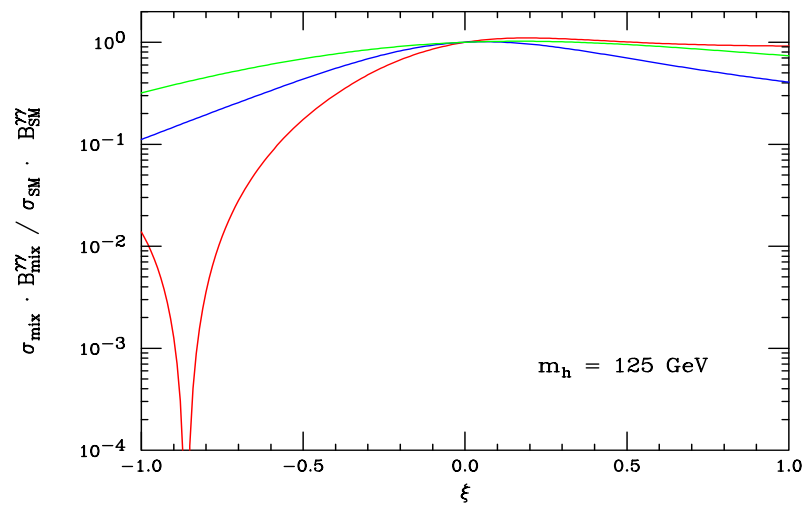

Figure 4: The ratio of production cross section times branching fraction for $p p \rightarrow h \rightarrow \gamma \gamma$ via gluon fusion with radion mixing to the SM expectations as a function of $\xi$. The Higgs mass is taken to be $125 \mathrm{GeV}$. The red (blue; green) curves correspond to the choice $m_{r}=300$ $\mathrm{GeV}, v / \Lambda=0.2(500,0.2 ; 300,0.1)$.

that if such a set of measurements were realized a large fraction of the parameter space would be excluded. Direct searches at LHC/LC would completely cover the lower portion of the remaining parameter space shown in the figure leaving only the high mass radion as a possibility under these circumstances.

So far we have only considered the case where the SM fermion are confined to the TeV brane. It is possible instead to place the SM gauge and fermion fields in the RS bulk which can lead to alterations in the radion couplings to these fields. Mixing with the Higgs could then lead to variations somewhat different than those discussed above. This possibility has been investigated and yields the results in Fig. 6 originates. Here we see the results analogous to those shown in the left panel of Fig. 1; note that in the case of bulk fields the expectations of the partial width shifts for fermions and massive vector fields are no longer degenerate. Qualitatively, however, the overall shifts in the Higgs boson couplings due to its mixing with the radion are found to be insensitive to whether or not the SM fields are in the RS bulk.

In summary, we see that Higgs-radion mixing, which is present in some extra dimensional scenarios, can have a substantial effect on the properties of the Higgs boson. These modifications affect the widths and branching fractions of Higgs decay into various final states, which in turn can alter the expectations for Higgs production at both LEP and the LHC. For some regions of the parameters the size of these width and branching fraction shifts may require the precision of a Linear Collider to study in detail. 


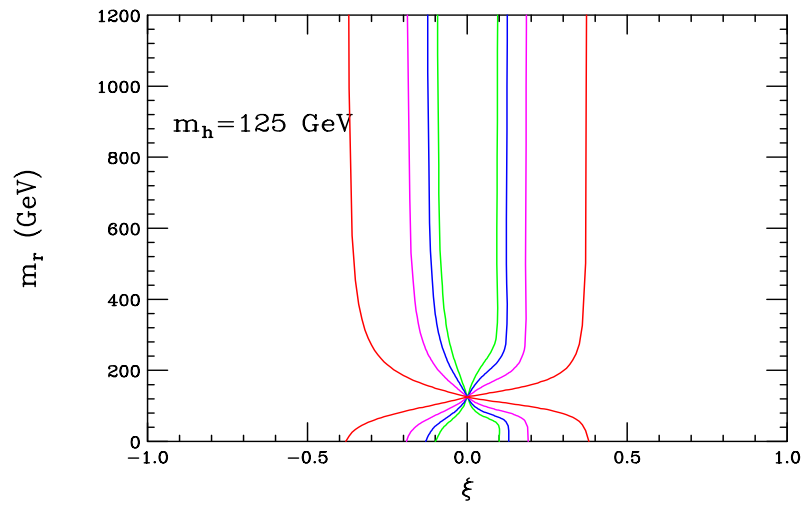

Figure 5: 95\% CL indirect bounds on the mass and mixing of the radion for a Higgs boson of mass $125 \mathrm{GeV}$ arising from precision measurements of the Higgs couplings at the LHC and LC. The allowed region lies between the corresponding vertical pair of curves. From inner to outer the curves correspond to values of $v / \Lambda=0.2,0.15,0.10$, and 0.05 , respectively.

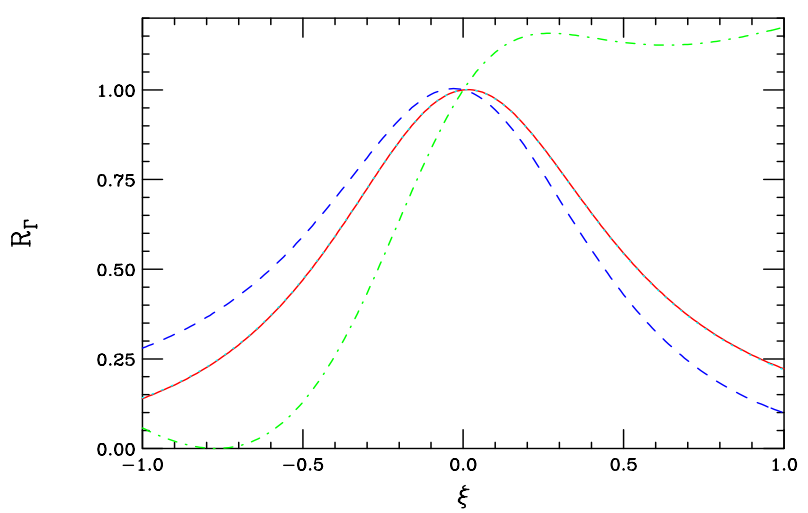

Figure 6: The effect of mixing on the partial widths of a $125 \mathrm{GeV}$ Higgs boson, described by the parameter $\xi$, assuming $v / \Lambda=0.2$ and a radion mass of $300 \mathrm{GeV}$ as discussed in the text. The solid(dash-dotted, dashed, dotted) corresponds to the $Z Z / W^{+} W^{-}(g g, \gamma \gamma, \bar{f} f)$ final states. 


\section{References}

1. For details of this analysis and original references, see J.L. Hewett and T.G. Rizzo, hep-ph/0202155 and 0207113. See also the talk by J. Gunion at this meeting. 